\title{
A Systematic Analysis of Literary Case Reports of Protein S Deficiency Implicated in Pathogenesis of Arterial Thrombosis
}

\author{
Pratyay Hasan ${ }^{1 *}$, Kazi Tuba-E Mozazfia ${ }^{2}$, Mohammed Abaye Deen Saleh ${ }^{3}$ and Dyuti Hasan ${ }^{4}$ \\ ${ }^{1}$ Department of Medicine, Dhaka Medical College Hospital, Dhaka, Bangladesh \\ ${ }^{2}$ Department of Medicine, Shaheed Suhrawardy Medical College, Dhaka, Bangladesh \\ ${ }^{3}$ Department of Cardiology, Dhaka Medical College Hospital, Dhaka, Bangladesh \\ ${ }^{4}$ Private Practitioner, Hatirpul Area, Dhaka, Bangladesh
}

Submission: April 28, 2017; Published: July 03, 2017

*Corresponding author: Pratyay Hasan, Indoor Medical Officer, Department of Medicine, Dhaka Medical College Hospital, Dhaka, Bangladesh, Email: phasanbd@gmail.com

\begin{abstract}
Introduction and background: Protein $\mathrm{S}$ is a vitamin $\mathrm{K}$ dependent natural anticoagulant. Deficiency of protein $\mathrm{S}$ is long known to cause venous thrombosis but not arterial thrombosis. Instances of arterial thrombosis due to protein S deficiency are very rare. So, very little can be known about the phenomenon, which is why this study attempts to elucidate on the patients who have suffered from the disease process.

Method: 24 articles describing 27 cases where arterial thrombosis occurred along with protein S deficiency were included and results from the reported cases were collated together then analyzed thoroughly.

Results: The minimum age was 4 and the maximum age was 64 , where the mean age was 37.07 ( \pm 16.88 SD) year, median age being 37 year. Cerebral arteries and peripheral arteries are the most commonly involved circulations (each constitute $29.63 \%$ of cases), making the coronary circulation the second most commonly involved region (14.81\%). The mean activity of protein $\mathrm{S}$ was $26( \pm 5 \mathrm{SE}) \%$, mean total protein $\mathrm{S}$ level was $52( \pm 10 \mathrm{SE}) \%$, mean free protein S level was $37.3( \pm 6.8 \mathrm{SE}) \%$, activity of protein C was $80( \pm 15 \mathrm{SE}) \%$, mean total protein C level was $74( \pm 11$ $\mathrm{SE}) \%$, mean anti-thrombin III level was $69( \pm 6 \mathrm{SE}) \%$.

Conclusion: Many circulations and regions in the body can be affected, most common being cerebral and peripheral, followed by coronary circulation. In the absence of standardized measurement and assay techniques of different forms of protein S, comparing and collating results is extremely difficult. A standard should be set up. And further larger studies should be undertaken to determine the true contribution of deficient protein $S$ level in pathogenesis of arterial thrombosis. Especially the researcher in hematology, cardiology and neurology should work together to determine the true significance of protein $\mathrm{S}$.
\end{abstract}

Keywords: Case series; Systematic analysis; Protein S; Arterial thrombosis; Cerebral infarct; Coronary infarct; Hematology; Cardiology; Neurology

\section{Introduction}

Protein S, a vitamin K dependent plasma glycoprotein was discovered in 1977 by Di Scipio et al. [1] in the city of Seattle, after which it was named [1,2]. The anticoagulant property of Protein $S$ has been discovered by Walker et al.[3] Hereditary deficiency of protein $\mathrm{S}$ has been long known for causing venous thrombosis but the effect of the deficiency in pathogenesis of arterial thrombosis is much less acknowledged [4,5]. It is because, thrombophilia is usually associated with venous, rather than arterial thrombosis. Since the first case of venous thromboembolism reported [6], it is known that a deficiency of protein $\mathrm{S}$ predisposes subjects to recurrent venous thromboembolism (VTE) and fetal loss [2].
But with advancement of time, accumulation of reported cases has raised the concern that arterial thrombosis can also occur due to protein S deficiency. Girolami et al. [7] have reported that both venous and arterial thrombosis may occur in any given patient with a thrombophilic state. Protein S deficiency has been reported as a suspected cause for arterial thrombosis in people under the age of 45 years [8]. Deficiency of any one of the Protein C, Protein S or Antithrombin III may predispose to in-situ thrombosis within either the arterial or venous system and are a cause of otherwise unexplained strokes in young patients [9]. Carod-Artal et al. [10] found that prothrombotic 
conditions are more frequent among the young ischaemic stroke patients classified as strokes of undetermined cause. Dykes et al. [11] have determined the prevalence of protein S deficiency to be between 0.03 and $0.13 \%$ in general population in a study involving $3788 \mathrm{Scottish}$ volunteers. The estimated prevalence in the general Japanese population is $1-2 \%$ [12]. But there is much scarcity of available literature documenting arterial thrombosis caused by protein S deficiency, which prompted this current study.

\section{Methodology}

For conduction of this study, a set of inclusion and exclusion criteria were determined.

\section{Inclusion criteria}

a) Article must be a case report, or letter to editor which will describe a case of arterial thrombosis.

b) In the reported case, protein S deficiency, alone or in combination with other abnormalities must be reported.

c) Article must be written in English.

d) Full text availability of the case report.

\section{Exclusion criteria}

A. Case report in other language.

B. Article types other than case report or letter to the editor, where a case is not reported.

C. Venous thrombosis.

D. Where protein S deficiency is not mentioned.

E. Reviews.

\section{Searching of literature}

A systemic search was conducted on 18th May 2016, in the electronic database "PubMed", www.ncbi.nlm.nih.gov/pubmed, using keywords, such as, "protein s", "case reports", "arterial", and "thrombosis". The exact search phrase is mentioned here: («protein s»[MeSH Terms] OR «protein s»[All Fields]) AND («case reports»[Publication Type] OR «case reports»[All Fields]) AND («arteries»[MeSH Terms] OR «arteries»[All Fields] OR «arterial»[All Fields]) AND («thrombosis»[MeSH Terms] OR «thrombosis»[All Fields]).

The search resulted with findings of total 94 articles, but again the search was refined with filters "case reports", and only articles with full text availability were sought, and ultimately 55 articles were found. Out of these, full texts of eight articles were not found, despite all efforts. Out of these eight case reports, three were in different language, so five remained, out of them, one was about venous thrombosis, one was about unrelated topic, so remained only four articles that were not found in total. Two personal case reports were also added, making the number of total available articles to 49 [13].

\section{Inclusion and exclusion of suitable reports}

But after thorough perusal of all the 49 articles, 25 articles were excluded. Out of these 25 articles, three were written in languages other than English, five mentioned venous thrombosis, four described anti-phospholipid antibody syndrome, one described anti-thrombin III deficiency, four mentioned isolated protein c deficiency, one mentioned factor $\mathrm{V}$ Leiden mutation, one mentioned beta-2-glycoprotein related arterial thrombosis, one was a comment on already included case report, and two were reviews, etc. only a total of 24 case reports were found that met the inclusion criteria. At last the analysis was conducted with these 24 articles [13-36]. These 24 articles described 27 cases where arterial thrombosis occurred along with protein $\mathrm{S}$ deficiency. The articles and the results are summarized in Table 1. The process is shown in Figure $1[37,38]$. For analysis of the studies, SPSS (Statistical Package for Social Science), version 23 (IBM Corporation) was used.

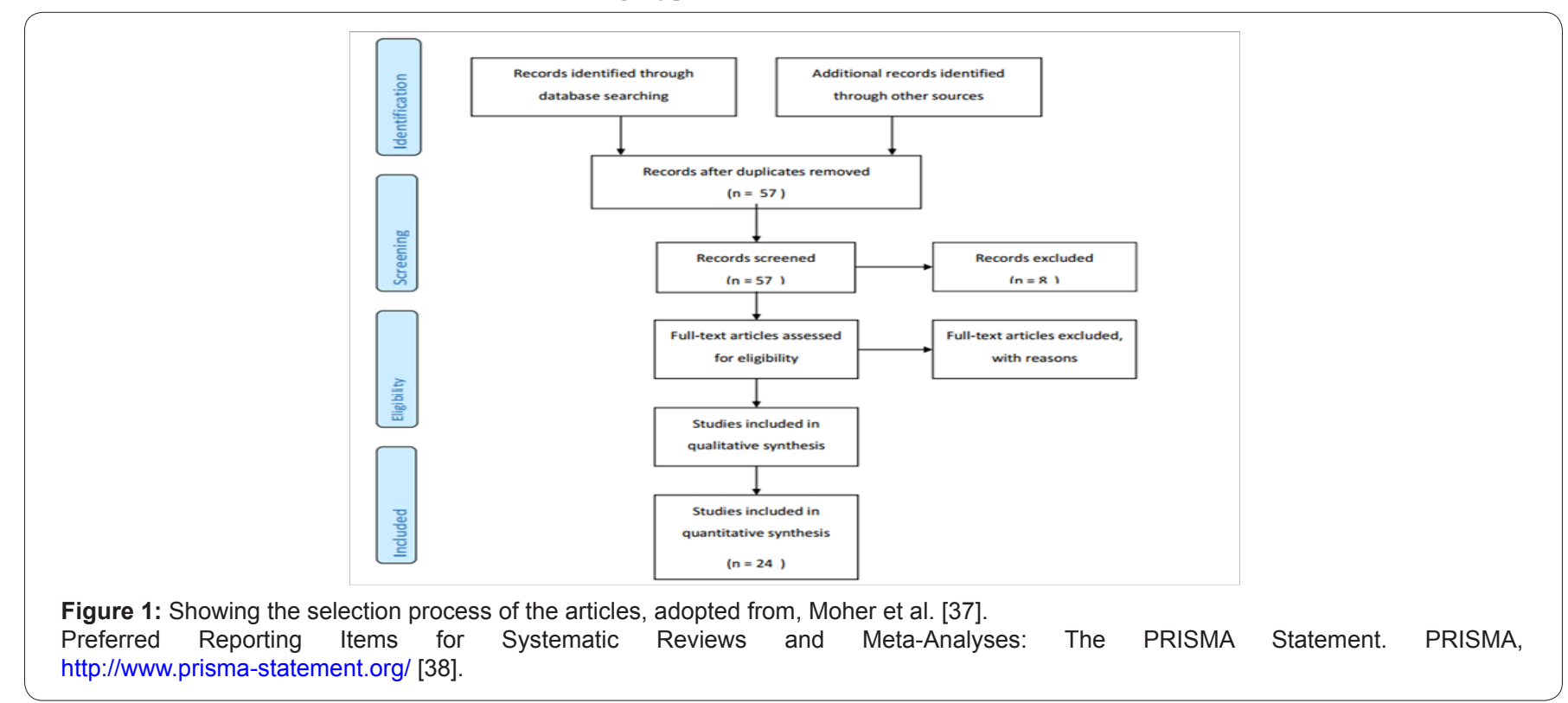




\begin{tabular}{|c|c|c|c|c|c|c|c|c|c|c|c|c|c|}
\hline 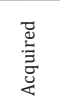 & 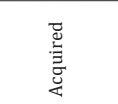 & & 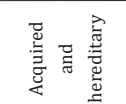 & & 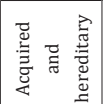 & 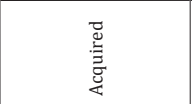 & & & & & & 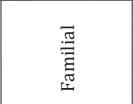 & \\
\hline 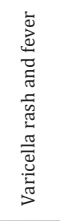 & 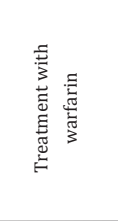 & & ثิ & & 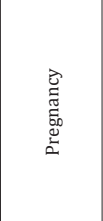 & 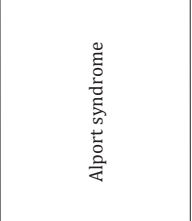 & & & & & & 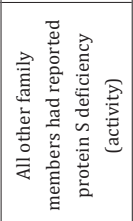 & \\
\hline & & 呇 & & 宧 & & & 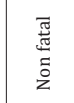 & 監 & & 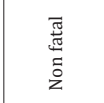 & & & \\
\hline & & $\ddagger$ & & $\approx$ & & & ま & & $\stackrel{0}{\circ}$ & & & 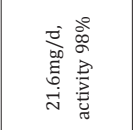 & \\
\hline $\begin{array}{l}\frac{\bar{w}}{\tilde{\Sigma}} \\
\frac{\bar{z}}{2}\end{array}$ & 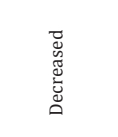 & 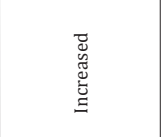 & 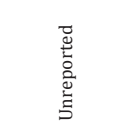 & 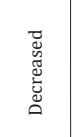 & 眔 & 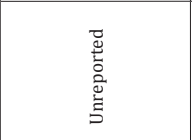 & 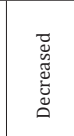 & 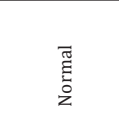 & 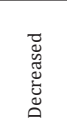 & 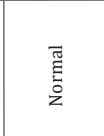 & 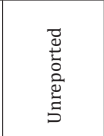 & $\stackrel{\overline{\widetilde{m}}}{\overline{\mathrm{g}}}$ & 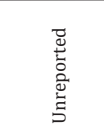 \\
\hline & $\delta$ & & & & & & & $\stackrel{\infty}{a}$ & & & & & \\
\hline$\stackrel{\sigma}{ }$ & $\hat{\omega}$ & & & ஜ & & i & 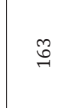 & $\stackrel{\infty}{\circ}$ & & & 8 & $\stackrel{\circ}{\circ}$ & 吕 \\
\hline 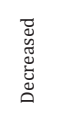 & 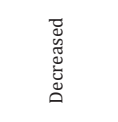 & 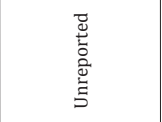 & 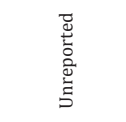 & 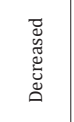 & 急 & 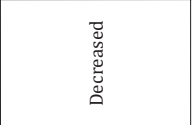 & 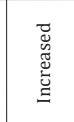 & 嵒 & $\frac{\overline{\underline{g}}}{\overline{\bar{z}}}$ & 覀 & 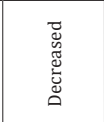 & 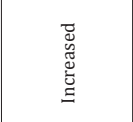 & 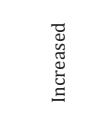 \\
\hline & & $\stackrel{\infty}{+}$ & & & สี่ & & ঋ & & & 吕 & $a$ & & \\
\hline & $\stackrel{m}{\circ}$ & in & & $\stackrel{\text { N }}{ }$ & & & $\begin{array}{l}\overline{\widetilde{m}} \\
\frac{\bar{\Sigma}}{2}\end{array}$ & $\stackrel{\Xi}{\Xi}$ & & & & & \\
\hline 今े & $\vec{\sim}$ & & 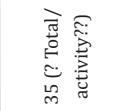 & & & 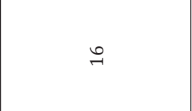 & & N & $\stackrel{\infty}{\sim}$ & & & $n$ & ㅇ \\
\hline 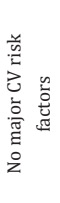 & 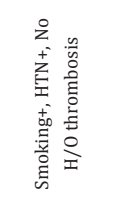 & 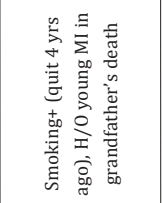 & 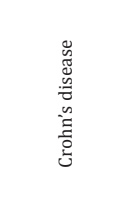 & 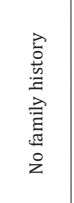 & & 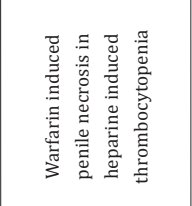 & 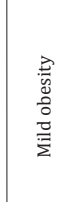 & 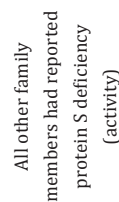 & & & & 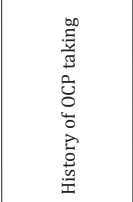 & 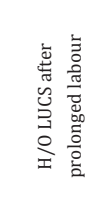 \\
\hline & 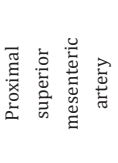 & 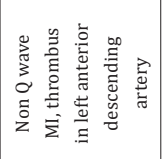 & 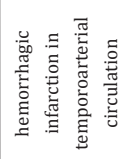 & 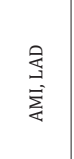 & 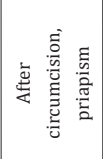 & 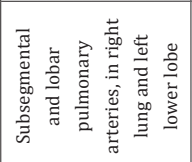 & 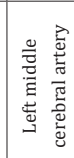 & 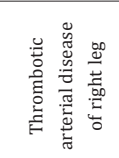 & 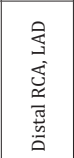 & 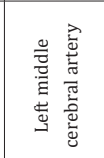 & 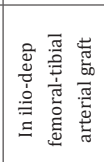 & 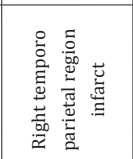 & 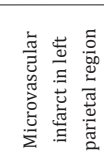 \\
\hline 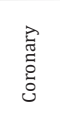 & 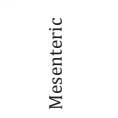 & 㴔 & 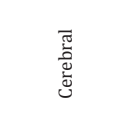 & 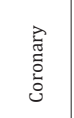 & 产 & 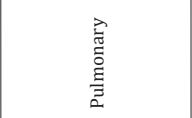 & 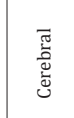 & 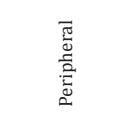 & 㴔 & चु్ & 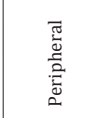 & 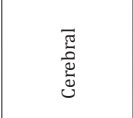 & 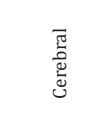 \\
\hline 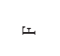 & 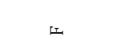 & $\Sigma$ & $\Sigma$ & w & $\Sigma$ & $\Sigma$ & I & $\Sigma$ & $\Sigma$ & $\Sigma$ & 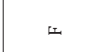 & 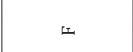 & $\omega$ \\
\hline$\infty$ & \& & $\stackrel{\text { ก }}{4}$ & $\stackrel{m}{m}$ & $\stackrel{n}{N}$ & $\sigma$ & ڤ & $\hat{m}$ & $\vec{\lambda}$ & ڤ & $\stackrel{\infty}{\circ}$ & t & $\stackrel{\infty}{\sim}$ & $\vec{N}$ \\
\hline 六 & : & S & ت्ञ & ర్ & ت्ञ & : & 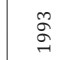 & 离 & 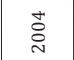 & $\vec{\sigma}$ & ثे & 芫 & 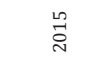 \\
\hline 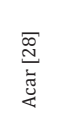 & 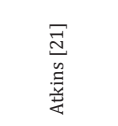 & 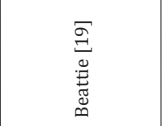 & 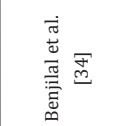 & 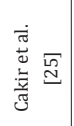 & 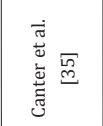 & 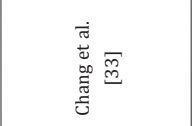 & 咅 & 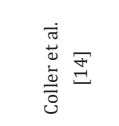 & 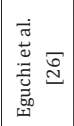 & 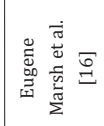 & 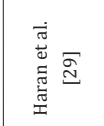 & 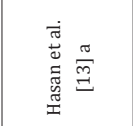 & 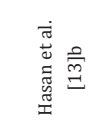 \\
\hline
\end{tabular}




\begin{tabular}{|c|c|c|c|c|c|c|c|c|c|c|c|c|}
\hline 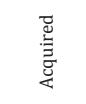 & 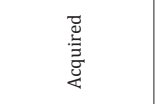 & & 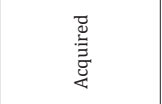 & 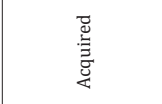 & & & 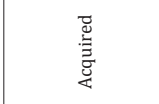 & & & & & 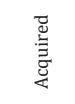 \\
\hline 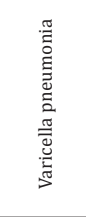 & 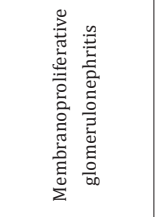 & & 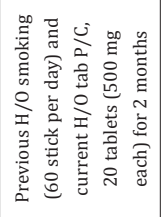 & 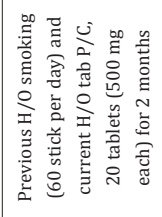 & & 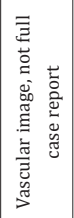 & 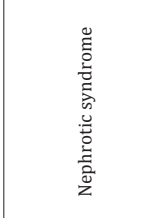 & & & & & 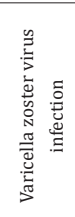 \\
\hline & & & & & $\begin{array}{l}\text { 愛 } \\
\text { 竞 }\end{array}$ & & & & & & & \\
\hline 8 & $\therefore$ & & $\stackrel{\circ}{\infty}$ & 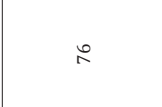 & ? & 8 & & & $\therefore$ & 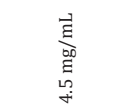 & $\stackrel{\Xi}{ }$ & \\
\hline 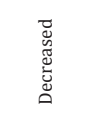 & 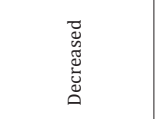 & 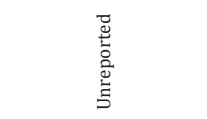 & 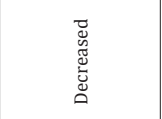 & 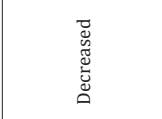 & 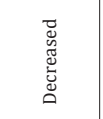 & 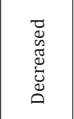 & 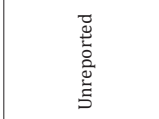 & 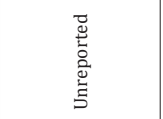 & $\begin{array}{l}\text { 穂 } \\
\text { 言 }\end{array}$ & 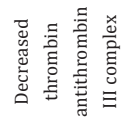 & 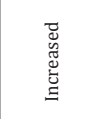 & 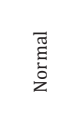 \\
\hline & & & & & 8 & ๖ & $\ddot{6}$ & & & & & \\
\hline & ๖ & & $\approx$ & \& & & & & & & & 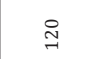 & \\
\hline 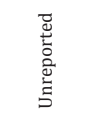 & 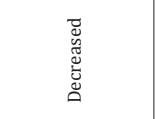 & 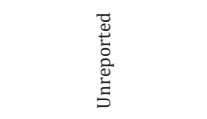 & 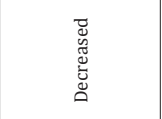 & 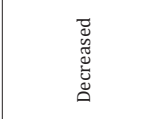 & 嵒 & 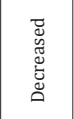 & 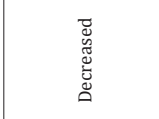 & 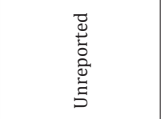 & 穂 & 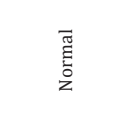 & 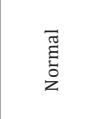 & $\begin{array}{l}\text { 臭 } \\
\frac{\mathrm{z}}{\mathrm{z}}\end{array}$ \\
\hline & & & & & & \pm & $\stackrel{\leftrightarrow \sim N}{N}$ & & & & q & \\
\hline & $\stackrel{\infty}{\sim}$ & 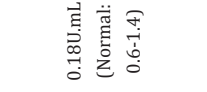 & & & 0 & $R$ & $\stackrel{\infty}{0}$ & & & & $\stackrel{n}{n}$ & \\
\hline & & & in & $m$ & & . & & $\stackrel{\infty}{\sim}$ & gे & $\hat{m}$ & & \\
\hline & 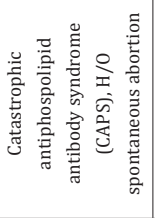 & 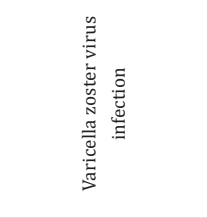 & 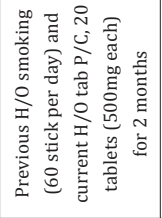 & 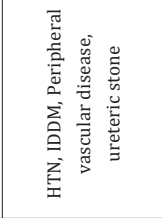 & & 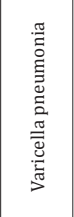 & 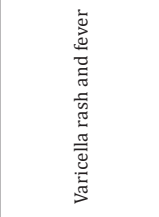 & & & 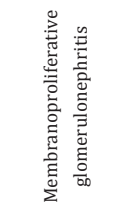 & & 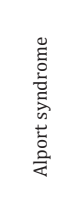 \\
\hline 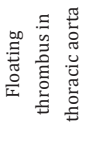 & 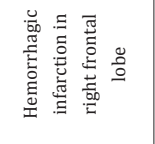 & 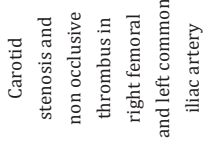 & 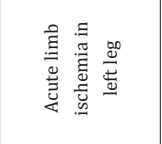 & 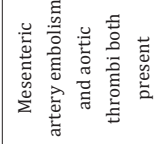 & 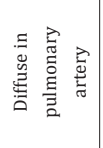 & 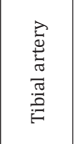 & 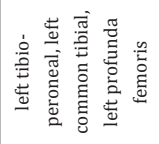 & 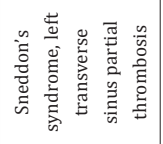 & 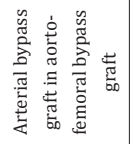 & 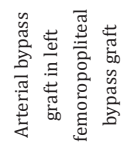 & 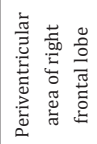 & 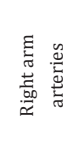 \\
\hline$\frac{\frac{5}{5}}{\frac{5}{4}}$ & 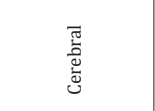 & 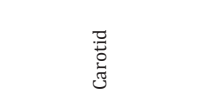 & 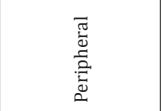 & 㺃 & 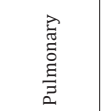 & 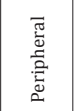 & 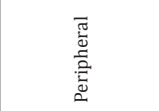 & 歌 & 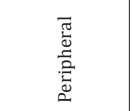 & 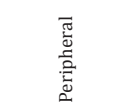 & 愛 & 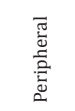 \\
\hline w & $w$ & $\Sigma$ & $\Sigma$ & w & $w$ & $\Sigma$ & $\Sigma$ & w & $\Sigma$ & $\Sigma$ & w & is \\
\hline$\approx$ & $\stackrel{\infty}{\infty}$ & ळे & \& & $\stackrel{\infty}{+}$ & n & f & $\hat{m}$ & $\ddot{m}$ & ก & $\vec{\sigma}$ & $\dot{m}$ & + \\
\hline 㐔 & 今े & 。ू & 今े & స్ & $\underset{\text { N }}{\text { N }}$ & $\begin{array}{l}\infty \\
\stackrel{\infty}{\sigma} \\
\sigma\end{array}$ & 离 & సี & S & S & 高 & 总 \\
\hline 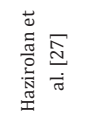 & 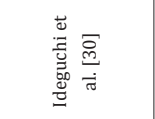 & 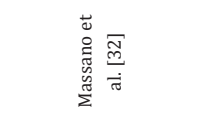 & 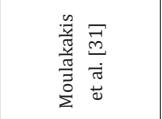 & 高 & 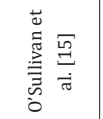 & 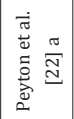 & 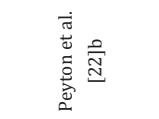 & 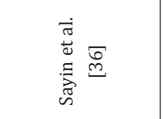 & 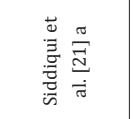 & 离竞 & 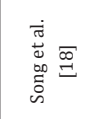 & 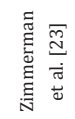 \\
\hline
\end{tabular}




\section{Results}

Summary of the cases and findings are shown in Table 1.

In this study a total of 27 cases are included, among them 13 are male (51.85\%) and 14 are female (48.15\%), as shown in Figure 2.

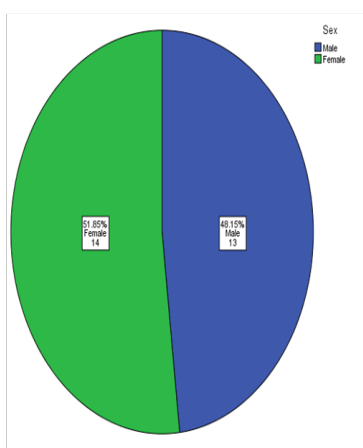

Figure 2: Sex distribution of cases.

The minimum age was 4 and the maximum age was 64 , where the mean age was $37.07( \pm 16.88$ SD) year, median age being 37 year (Figure $3 \& 4$ ) and (Table 2).

Table 2: Age distribution of cases.

\begin{tabular}{|c|c|c|c|c|}
\hline & $\begin{array}{c}\text { Mean } \\
\text { (Year) }\end{array}$ & $\begin{array}{c}\text { Standard } \\
\text { Deviation }\end{array}$ & $\begin{array}{c}\text { Median } \\
\text { Age }\end{array}$ & $\begin{array}{c}\text { Number of } \\
\text { Cases }\end{array}$ \\
\hline Male & 39.85 & 15.27 & 39 & 13 \\
\hline Female & 34.5 & 18.44 & 35 & 14 \\
\hline Both sex & 37.07 & 16.88 & 37 & 27 \\
\hline
\end{tabular}

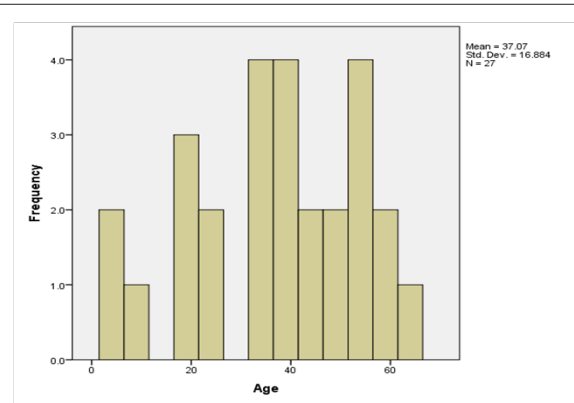

Figure 3: Age distribution of the cases.

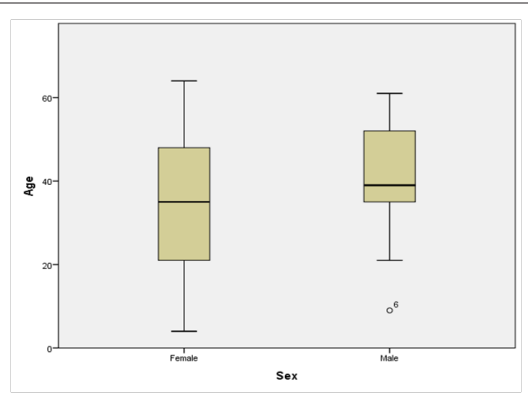

Figure 4: Age and sex distribution of cases.
The arterial circulation involved are shown in Figure 5, demonstrating the fact that cerebral arteries and peripheral arteries are the most common circulations (each constitute $29.63 \%$ of cases), making the coronary circulation the second mostcommonly involved region (14.81\%). Figure 6 demonstrates the percentages of involved areas, grouped by sex. Cerebral circulation was most commonly involved in females $(22.22 \%)$ and peripheral circulation was most commonly involved in male $(22.22 \%)$.
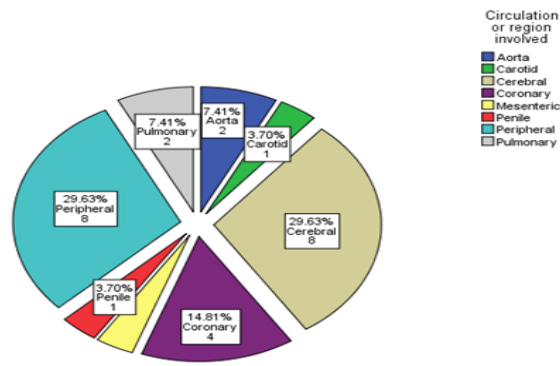

Figure 5: Regions involved.

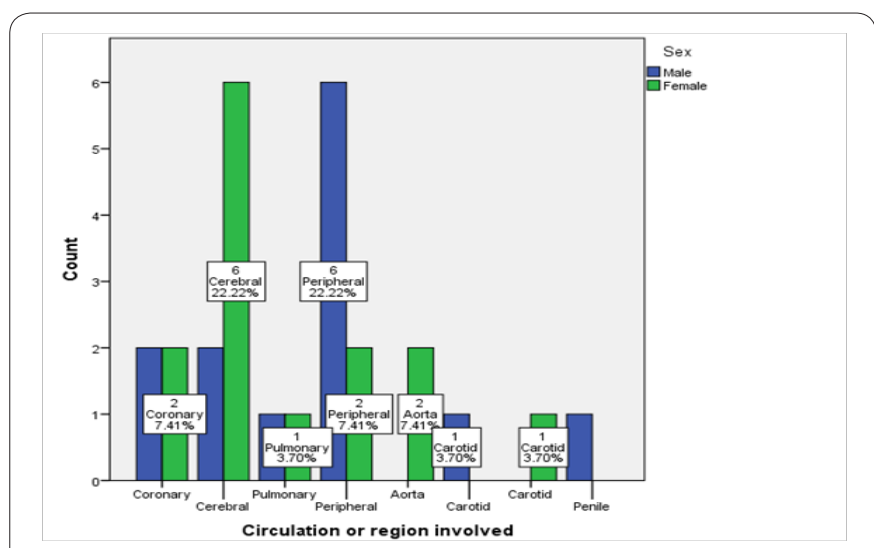

Figure 6: Involved regions in different sexes.

Among the 27, three cases (10\%) were fatal. The different measurements of protein S, protein C, and anti-thrombin III are shown in Table 3 and the same measurements according to sex distribution are shown in Table 4.

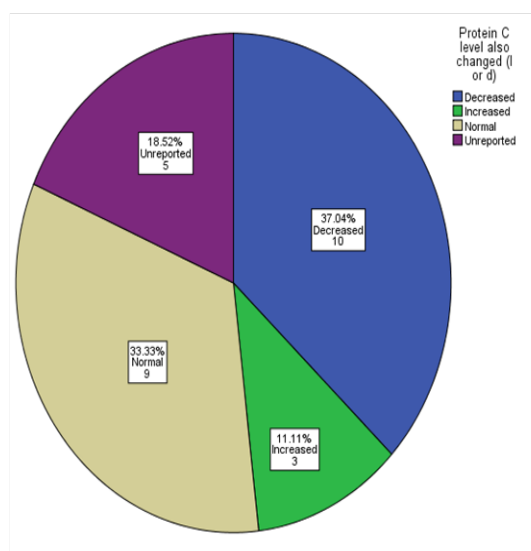

Figure 7: Change in protein $\mathrm{C}$ level 
Table 3: Measurement values of protein S, C and antithrombin III.

\begin{tabular}{|c|c|c|c|c|c|c|}
\hline & Count & Minimum & Maximum & Mean & $\begin{array}{c}\text { Standard Error } \\
\text { of Mean }\end{array}$ & Median \\
\hline $\begin{array}{c}\text { Protein S level } \\
\text { Activity in } \\
\text { percentage }\end{array}$ & 27 & 3 & 59 & 26 & 5 & 22 \\
\hline $\begin{array}{l}\text { Protein S level } \\
\text { total in percentage }\end{array}$ & 27 & 6 & 102 & 52 & 10 & 57 \\
\hline $\begin{array}{l}\text { Protein S level free } \\
\text { in percentage }\end{array}$ & 27 & 9.0 & 62.2 & 37.3 & 6.8 & 42.5 \\
\hline $\begin{array}{c}\text { Protein C level } \\
\text { Activity in } \\
\text { percentage }\end{array}$ & 27 & 19 & 169 & 80 & 15 & 63 \\
\hline $\begin{array}{l}\text { Protein C level } \\
\text { total in percentage }\end{array}$ & 27 & 45 & 103 & 74 & 11 & 63 \\
\hline $\begin{array}{c}\text { Protein C level } \\
\text { free in percentage }\end{array}$ & 27 & . & . & . & . & . \\
\hline $\begin{array}{l}\text { Antithrombin III } \\
\text { level in percentage }\end{array}$ & 27 & 36 & 121 & 69 & 6 & 70 \\
\hline
\end{tabular}

Table 4: Measurements of protein S, C and antithrombin III in different sex groups.

\begin{tabular}{|c|c|c|c|c|c|c|c|c|c|c|c|c|}
\hline & \multicolumn{6}{|c|}{ Male } & \multicolumn{6}{|c|}{ Female } \\
\hline & Count & Minimum & Maximum & Mean & $\begin{array}{c}\text { Standard } \\
\text { Error of } \\
\text { Mean }\end{array}$ & Median & Count & Minimum & Maximum & Mean & $\begin{array}{l}\text { Standard } \\
\text { Error of } \\
\text { Mean }\end{array}$ & Median \\
\hline $\begin{array}{c}\text { Protein S } \\
\text { level Activity } \\
(\%)\end{array}$ & 13 & 16 & 59 & 32 & 7 & 30 & 14 & 3 & 50 & 20 & 7 & 16 \\
\hline $\begin{array}{c}\text { Protein S } \\
\text { level total (\%) }\end{array}$ & 13 & 57 & 102 & 74 & 10 & 69 & 14 & 6 & 75 & 34 & 11 & 28 \\
\hline $\begin{array}{c}\text { Protein S } \\
\text { level free }(\%)\end{array}$ & 13 & 14.0 & 62.2 & 40.8 & 9.2 & 48.0 & 14 & 9.0 & 45.0 & 31.3 & 11.3 & 40.0 \\
\hline $\begin{array}{c}\text { Protein C } \\
\text { level Activity } \\
(\%)\end{array}$ & 13 & 20 & 98 & 47 & 26 & 23 & 14 & 19 & 169 & 90 & 17 & 65 \\
\hline $\begin{array}{c}\text { Protein C } \\
\text { level total (\%) }\end{array}$ & 13 & 45 & 103 & 70 & 17 & 63 & 14 & 60 & 99 & 80 & 19 & 80 \\
\hline $\begin{array}{c}\text { Protein C } \\
\text { level free (\%) }\end{array}$ & 13 & . & . & . & . & . & 14 & . & . & . & . & . \\
\hline $\begin{array}{l}\text { Antithrombin } \\
\text { III level (\%) }\end{array}$ & 13 & 36 & 76 & 57 & 8 & 60 & 14 & 44 & 121 & 77 & 8 & 73 \\
\hline
\end{tabular}

Table 5: Changes in protein $\mathrm{C}$ and anti-thrombin III level

\begin{tabular}{|c|c|c|c|c|c|c|c|c|c|c|c|}
\hline \multirow{9}{*}{$\begin{array}{c}\text { Anti } \\
\text { thrombin } \\
\text { III level } \\
\text { also } \\
\text { changed }\end{array}$} & & \multicolumn{10}{|c|}{ Protein C level also changed ( $I$ or $d$ ) } \\
\hline & & \multirow[b]{2}{*}{ Count } & \multirow[b]{2}{*}{$\begin{array}{l}\text { (\% of } \\
\text { total) }\end{array}$} & \multirow{2}{*}{$\begin{array}{c}\text { Decreased } \\
\text { Count }\end{array}$} & \multirow[b]{2}{*}{$\begin{array}{l}\text { (\% of } \\
\text { total) }\end{array}$} & \multicolumn{2}{|c|}{ Increased } & \multicolumn{2}{|c|}{ Normal } & \multicolumn{2}{|c|}{ Unreported } \\
\hline & & & & & & Count & $\begin{array}{l}\text { (\% of } \\
\text { total) }\end{array}$ & Count & $\begin{array}{l}\text { (\% of } \\
\text { total) }\end{array}$ & Count & $\begin{array}{l}\text { (\% of } \\
\text { total) }\end{array}$ \\
\hline & & 3 & 10.0 & 0 & 0.0 & 0 & 0.0 & 0 & 0.0 & 0 & 0.0 \\
\hline & Decreased & 0 & 0.0 & 6 & 20.0 & 1 & 3.3 & 2 & 6.7 & 1 & 3.3 \\
\hline & $\begin{array}{l}\text { Decreased } \\
\text { thrombin } \\
\text { antithrombin } \\
\text { III complex }\end{array}$ & 0 & 0.0 & 0 & 0.0 & 0 & 0.0 & 1 & 3.3 & 0 & 0.0 \\
\hline & Increased & 0 & 0.0 & 0 & 0.0 & 0 & 0.0 & 1 & 3.3 & 1 & 3.3 \\
\hline & Normal & 0 & 0.0 & 1 & 3.3 & 1 & 3.3 & 5 & 16.7 & 0 & 0.0 \\
\hline & Unreported & 0 & 0.0 & 3 & 10.0 & 1 & 3.3 & 0 & 0.0 & 3 & 10.0 \\
\hline
\end{tabular}


In Table 5 and Figure 7 associated changes in protein $\mathrm{C}$ and anti-thrombin III levels are shown. Although in some patients, protein C levels are not reported (5 cases, $18.52 \%$ ), but in most of the patients, protein $\mathrm{C}$ level was decreased $(37.04 \%)$. There is a rather interesting rise in protein $\mathrm{C}$ level in $11.11 \%$ of cases.

\section{Discussion}

From the accumulated results, it can be seen that there is not much difference in distribution of sex of the cases. The age distribution is also a similar tale, as it can happen in very early years (4 years) to very late years (64 years), in both sex groups (Figure 4) and (Table 2).

The arterial circulation involved are shown in Figure 5, demonstrating the fact that cerebral arteries and peripheral arteries are the most common circulations (each constitute $29.63 \%$ of cases), making the coronary circulation the second mostcommonly involved region (14.81\%). Figure 5 demonstrates the percentages of involved areas, grouped by sex. Cerebral circulation was most commonly involved in females $(22.22 \%)$ and peripheral circulation was most commonly involved in male $(22.22 \%)$. This phenomenon should be investigated further in large studies. The result shows that not only cerebral circulation, peripheral circulation and coronary circulation are also potentially at risk of arterial thrombosis in case of protein $S$ deficiency. Maybe the effect and distribution of measurements of protein S levels should be investigated in cases of myocardial infarctions, especially, if traditional risk factors are absent and uncommon causes are sought.

In the included case reports, protein $\mathrm{S}$ are measured in various forms, total antigen, free antigen (unbound) and functional activity and also, there are substantial amount of variability in expressing the results [13-36]. The cause behind this is there are two forms of protein $\mathrm{S}$ in plasma, a free form and a complex form. The free form of protein $\mathrm{S}$ is active. The bound form comprises $65 \%$ of the total protein $\mathrm{S}$, and it is complexed to $\mathrm{C} 4 \mathrm{~b}$-binding protein ( $\mathrm{C} 4 \mathrm{bBP}$ ) and is inactive. The availability of $\mathrm{C} 4 \mathrm{bBP}$ regulates the proportion of the free and bound forms of protein S. Due to this variability in the forms of protein $S$ in plasma, at least three types of hereditary Protein S deficiency mentioned in available literature: [39]

a. Type I (quantitative defect): In this classic type of protein S deficiency, all antigenic type of protein $\mathrm{S}$ level (total, free and bound) are decreased and so is the functional activity level. $[6,39,40]$.

b. Type II (qualitative defect): In this type of deficiency, all antigen levels of protein $\mathrm{S}$ are normal but functional activity is decreased.

c. Type III or Type IIa: In this type of deficiency, only free protein $\mathrm{S}$ level is decreased, and bound protein $\mathrm{S}$ level is increased, total amount of protein $S$ remains unaltered and within normal limit. Functional activity is decreased.
So, protein S functional activity is decreased in all types. It can be seen that type II deficiencies are not detected by free protein S antigen assays, and type II and type III deficiencies are not detected by total protein S antigen assays [39]. It is shown that some total protein $\mathrm{S}$ assay results could be normal even in the presence of a type I deficiency.

There are two types of assays that test for protein $S$ deficiency:

1. Functional (activity) assays.

2. Antigenic (quantitative immunologic) assays.

In the included studies, the authors have used different reference values. Actually protein $\mathrm{S}$ should be measured against age specific reference values. Dykes et al. [11] have proposed such reference values. In general, levels of total or free protein $\mathrm{S}$ antigen $<60$ to $65 \mathrm{IU} / \mathrm{dL}$ are considered to be in the deficient range [41]. Although almost every study included has reported their values in percentages, and the detailed is not known about any standard against which the percentage was determined. In the included studies not only the activity level but also the antigen level was also expressed in percentages. In a population study involving 3788 healthy Scottish volunteers, Dykes et al. [11] used $100 \%$ to denote the antigen present in $1 \mathrm{ml}$ of normal blood plasma derived from a group of 14 men and 11 women with age ranging between 21-62 years. Similar standards are used in different laboratories, but the detail of the standard are often unavailable to the clinicians, and so often absent in the reported case. But to analyze and accumulate the results together it was imperative that all results must be expressed in uniform measurement standards. Using international units could be a better alternative and could make comparison and collection of results a lot easier. In the current study, original values as reported by the authors of the case reports are retained.

Due to such variability in the forms of both protein $S$ and protein $\mathrm{C}$, reporting of the levels of protein $\mathrm{S}$ and protein $\mathrm{C}$ is a very much difficult task, as Marlar \& Gausman [42] have rightly described as "nightmare". In the included studies also, proof in favor of this statement is seen. Many have reported various combinations of measurements of activity level, total antigenic level, free antigenic levels of protein S and protein C. Some have reported antithrombin III level as well.

Protein S deficiency can also be acquired due to [2]

i. Vitamin K-antagonist therapy.

ii. Oral contraceptives.

iii. Pregnancy.

iv. Various disorders, such as

a. Liver disease.

b. Nephritic syndrome, 
c. Disseminated intravascular coagulation and

d. Chronic infections (e.g. HIV).

In many case reports included in this study, co-existent protein $\mathrm{C}$ deficiency is also reported. Heterozygous deficiency of protein $\mathrm{C}$ may be of two types, based on immunologic and functional assays:

a) Type 1 deficiency: Protein $\mathrm{C}$ concentration is approximately $50 \%$ of normal in both immunologic and functional assays [43]. Most genetic protein C mutations result in Type 1 deficiency [44].

b) Type 2 deficiency: Normal plasma protein $\mathrm{C}$ antigen levels with decreased functional activity $[45,46]$. This type constitutes for $15 \%$ of symptomatic deficiencies $[47,48]$.

To detect these two types of protein $\mathrm{C}$ defects, two types of tests are employed, which are:

1. Immunologic: Antigen level is measured using any one of the following [49]:

a) Electroimmuno assay.

b) Enzyme linked immunosorbent assay, or

c) Radioimmunoassay.

2. Functional assays [50-52]:

a. Measuring amidolytic activity of protein $\mathrm{C}$ in chromogenic assays: chromogenic tests to see amidolytic activity have better performance than clotting assays, and is preferred by routine laboratories.

b. Measuring anticoagulant activity in clotting assays: occasionally they may detect Type 2 defects that are missed in chromogenic tests measuring amidolytic activity [52].

Functional assays are preferred generally because they detect both Type 1 and Type 2 defect [53]. But immunologic assays help to distinguish between Type 1 and Type 2 protein $\mathrm{C}$ deficiency.

\section{Conclusion}

There is not much interesting information in age and sex distribution of protein S deficient patients who presented with arterial thrombosis. Many circulations and regions in the body can be affected, most common being cerebral and peripheral, followed by coronary circulation. In the absence of standardized measurement and assay techniques of different forms of protein $\mathrm{S}$, comparing and collating results is extremely difficult. A standard should be set up. And further larger studies should be undertaken to determine the true contribution of deficient protein $S$ level in pathogenesis of arterial thrombosis. Especially the researcher in hematology, cardiology and neurology should work together to determine the true significance of protein S.

\section{References}

1. Di Scipio RG, Hermodson MA, Yates SG, Davie EW (1977) A comparison of human prothrombin, factor IX (Christmas factor), factor X (Stuart factor), and protein S. Biochemistry 16(4): 698-706.

2. ten Kate MK, van der Meer J (2008) Protein S deficiency: a clinical perspective. Haemophilia 14(6): 1222-1228.

3. Walker FJ (1980) Regulation of activated protein C by a new protein. A possible function for bovine protein S. J Biol Chem 255(12): 55215524.

4. De Stefano V, Leone G, Mastrangelo S, Tripodi A, Rodeghiero F, et al. (1994) Clinical manifestations and management of inherited thrombophilia: retrospective analysis and follow-up after diagnosis of 238 patients with congenital deficiency of antithrombin III, protein C, protein S. Thromb Haemost 72(3): 352-358.

5. Esmon CT (1989) The roles of protein C and thrombomodulin in the regulation of blood coagulation. J Biol Chem 264(9): 4743-4746.

6. Schwarz HP, Fischer M, Hopmeier P, Batard MA, Griffin JH (1984) Plasma protein $\mathrm{S}$ deficiency in familial thrombotic disease. Blood 64(6): 1297-1300.

7. Girolami A, Simioni P, Scarano L, Girolami B (1997) Venous and arterial thrombophilia. Haematologica 82(1): 96-100.

8. Allaart CF, Aronson DC, Ruys T, Rosendaal FR, van Bockel JH, et al. (1990) Hereditary protein S deficiency in young adults with arterial occlusive disease. Thromb Haemost 64(2): 206-210.

9. Ropper AH, Samuels MA (2009) Cerebrovascular disease. Adams Victors Princ Neurol ( $9^{\text {th }}$ Edn.), McGraw Hill Professional, USA, p. 752.

10. Carod-Artal FJ, Nunes SV, Portugal D, Silva TVF, Vargas AP (2005) Ischemic stroke subtypes and thrombophilia in young and elderly Brazilian stroke patients admitted to a rehabilitation hospital. Stroke 36(9): 2012-2014.

11. Dykes AC, Walker ID, McMahon AD, Islam SI, Tait RC (2001) A study of Protein S antigen levels in 3788 healthy volunteers: influence of age, sex and hormone use, and estimate for prevalence of deficiency state. Br J Haematol 113(3): 636-641.

12. Miyata T, Kimura R, Kokubo Y, Sakata T (2006) Genetic risk factors for deep vein thrombosis among Japanese: importance of protein S K196E mutation. Int J Hematol 83(3): 217-223.

13. Hasan P, Chowdhury S, Kabir A, Deb SR, Hossain M, et al. (2015) Two Cases of Young Females Suffering From Stroke with Protein S Deficiency. Bangladesh J Med 26(1): 39-42.

14. Coller BS, Owen J, Jesty J, Horowitz D, Reitman MJ, et al. (1987) Deficiency of plasma protein S, protein $\mathrm{C}$, or antithrombin III and arterial thrombosis. Arteriosclerosis 7(5): 456-462.

15. O’Sullivan J, Chatuverdi R, Bennett MK, Hunter S (1992) Protein S deficiency: early presentation and pulmonary hypertension. Arch Dis Child 67(7): 960-961.

16. Marsh EE, Biller J, Adams HP, Kaplan JM (1991) Cerebral infarction in patients with nephrotic syndrome. Stroke 22(1): 90-93.

17. Chaturvedi S (1993) Fulminant cerebral infarctions with membranous nephropathy. Stroke 24(3): 473-475.

18. Song KS, Won DI, Lee AN, Kim CH, Kim JS (1994) A case of nephrotic syndrome associated with protein $S$ deficiency and cerebral thrombosis. J Korean Med Sci 9(4): 347-350.

19. Beattie S, Norton M, Doll D (1997) Coronary thrombosis associated with inherited protein S deficiency: a case report. Heart Lung 26(1): 76-79. 
20. Siddiqi FA, Tepler J, Fantini GA (1997) Acquired protein S and antithrombin III deficiency caused by nephrotic syndrome: an unusual cause of graft thrombosis. J Vasc Surg 25(3): 576-580.

21. Atkins KA, Zehnder JL (1998) Mesenteric artery thrombosis: a case report of combined protein $\mathrm{S}$ and protein $\mathrm{C}$ deficiency. Am J Hematol 58(3): 246-247.

22. Peyton BD, Cutler BS, Stewart FM (1998) Spontaneous tibial artery thrombosis associated with varicella pneumonia and free protein $\mathrm{S}$ deficiency. J Vasc Surg 27(3): 563-567.

23. Zimmerman AA, Watson RS, Williams JK (1999) Protein S deficiency presenting as an acute postoperative arterial thrombosis in a fouryear-old child. Anesth Analg 88(3): 535-537.

24. Onwuanyi A, Sachdeva R, Hamirani K, Islam M, Parris R (2001) Multiple aortic thrombi associated with protein $\mathrm{C}$ and $\mathrm{S}$ deficiency. Mayo Clin Proc 76(3): 319-322.

25. Cakir O, Ayyildiz O, Oruc A, Eren N (2002) A young adult with coronary artery and jugular vein thrombosis: a case report of combined protein $\mathrm{S}$ and protein C deficiency. Heart Vessels 17(2): 74-76.

26. Eguchi K, Ohtaki E, Misu K, Aikawa M, Sumiyoshi T, et al. (2004) Acute myocardial infarction caused by embolism of thrombus in the right coronary sinus of Valsalva: a case report and review of the literature. J Am Soc Echocardiogr 17(2): 173-177.

27. Hazirolan T, Perler BA, Bluemke DA (2004) Floating thoracic aortic thrombus in "protein S" deficient patient. J Vasc Surg 40(2): 381.

28. Acar G, Dogan A, Altinbas A, Turker Y (2006) Recurrent acute stent thrombosis associated with protein $\mathrm{C}$ and $\mathrm{S}$ deficiencies. Int $\mathrm{J}$ Cardiovasc Imaging 22(3-4): 333-337.

29. Haran MZ, Lichman I, Berebbi A, Weinmann E, Rosenberg N (2007) Unbalanced protein $S$ deficiency due to warfarin treatment as a possible cause for thrombosis. Br J Haematol 139(2): 310-311.

30. Ideguchi H, Ohno S, Ueda A, Ishigatsubo Y (2007) Catastrophic antiphospholipid syndrome associated with malignancies (case report and review of the literature). Lupus 16(1): 59-64.

31. Moulakakis KG, Sfyroeras G, Pavlidis P, Besias N, Maras D, et al. (2007) Hypercoagulable state due to alcohol-paracetamol syndrome producing acute limb ischemia. Vasc Endovascular Surg 41(4): 362365.

32. Massano J, Ferreira D, Toledo T, Mansilha A, Azevedo E, et al. (2008) Stroke and multiple peripheral thrombotic events in an adult with varicella. Eur J Neurol 15(10): e90-e91.

33. Chang IH, Ha MS, Chi BH, Kown YW, Lee SJ (2010) Warfarin-induced penile necrosis in a patient with heparin-induced thrombocytopenia. J Korean Med Sci 25(9): 1390-1393.

34. Benjilali L, Aidi S, El Mansouri H, Benabdejlil M, Jiddane M, et al. (2011) Cerebral thrombosis complicating Crohn's disease: two cases. J Stroke Cerebrovasc Dis 20(6): 565-569.

35. Canter HI, Coskuner ER (2011) Penile necrosis due to priapism developed after circumcision in a patient with protein S deficiency. J Sex Med 8(11): 3236-32340.

36. Sayin MR, Akpinar I, Karabag T, Aydin M, Dogan SM, et al. (2012) Left Main Coronary Artery Thrombus Resulting from Combined Protein C and S Deficiency. Intern Med 51(21): 3041-3044.
37. Moher D, Liberati A, Tetzlaff J, Altman DG, Group TP (2009) Preferred Reporting Items for Systematic Reviews and Meta-Analyses: The PRISMA Statement. PLOS Med 6(7): e1000097.

\section{8. (2016) PRISMA.}

39. Van Cott EM, Ledford-Kraemer M, Meijer P, Nichols WL, Johnson SM, et al. (2005) Protein S Assays: An Analysis of North American Specialized Coagulation Laboratory Association Proficiency Testing. Am J Clin Pathol 123(5): 778-785.

40. Broekmans AW, Bertina RM, Reinalda-Poot J, Engesser L, Muller HP, et al. (1985) Hereditary protein $S$ deficiency and venous thromboembolism. A study in three Dutch families. Thromb Haemost 53(2): 273-277.

41. Lijfering WM, Mulder R, ten Kate MK, Veeger NJGM, Mulder AB, et al. (2009) Clinical relevance of decreased free protein $S$ levels: results from a retrospective family cohort study involving 1143 relatives. Blood 113(6): 1225-1230.

42. Marlar RA, Gausman JN (2011) Protein S abnormalities: A diagnostic nightmare. Am J Hematol 86(5): 418-421.

43. Broekmans A, Bertina R (1985) Protein C. In: Recent Advances in Blood Coagulation. Poller L (ed.), Churchill Livingstone, New York, USA, p. 117.

44. Goldenberg NA, Manco-Johnson MJ (2008) Protein C deficiency. Haemophilia 14(6): 1214-1221.

45. Reitsma PH, Bernardi F, Doig RG, Gandrille S, Greengard JS, et al. (1995) Protein C deficiency: a database of mutations, 1995 update. On behalf of the Subcommittee on Plasma Coagulation Inhibitors of the Scientific and Standardization Committee of the ISTH. Thromb. Haemost 73(5): 876-889.

46. Lind B, Johnsen AH, Thorsen S (1997) Naturally occurring Arg(-1) to His mutation in human protein $\mathrm{C}$ leads to aberrant propeptide processing and secretion of dysfunctional protein C. Blood 89(8): 2807-2816.

47. Dahlbäck B (1995) The protein C anticoagulant system: inherited defects as basis for venous thrombosis. Thromb Res 77(1): 1-43.

48. Bertina RM (2000) Protein C deficiency and venous thrombosis--the search for the second genetic defect. Thromb Haemost 83(3): 360-361.

49. Michiels JJ, Hamulyák K (1998) Laboratory diagnosis of hereditary thrombophilia. Semin Thromb Hemost 24(4): 309-320.

50. Comp PC, Nixon RR, Esmon CT (1984) Determination of functional levels of protein $\mathrm{C}$, an antithrombotic protein, using thrombinthrombomodulin complex. Blood 63(1): 15-21.

51. Bertina RM, Broekmans AW, Krommenhoek-van Es C, van Wijngaarden A (1984) The use of a functional and immunologic assay for plasma protein $\mathrm{C}$ in the study of the heterogeneity of congenital protein $\mathrm{C}$ deficiency. Thromb Haemost 51(1): 1-5.

52. Vigano D’Angelo S, Comp PC, Esmon CT, D’Angelo A (1986) Relationship between protein $\mathrm{C}$ antigen and anticoagulant activity during oral anticoagulation and in selected disease states. J Clin Invest 77(2): 416 425.

53. Foy P, Moll S (2009) Thrombophilia: 2009 update. Curr Treat Options Cardiovasc Med 11(2): 114-128. 


\section{Your next submission with Juniper Publishers} will reach you the below assets

- Quality Editorial service

- Swift Peer Review

- Reprints availability

- E-prints Service

- Manuscript Podcast for convenient understanding

- Global attainment for your research

- Manuscript accessibility in different formats ( Pdf, E-pub, Full Text, Audio)

- Unceasing customer service

Track the below URL for one-step submission https://juniperpublishers.com/online-submission.php 